\section{[gw22-e0103] CLOPIDOGREL RESPONSE VARIABILITY AND ITS CORRELATION WITH RECURRENT CARDIOVASCULAR EVENTS IN CHINESE PATIENTS UNDERGOING PERCUTANEOUS CORONARY INTERVENTION}

Liu Yamin, Liu Naifeng, Li Weilan, Shao Hua, Zhi Hong Zhongda Hospital Affiliated To Southeast University, Nanjing, China

\subsection{6/heartjn|-2011-300867.330}

Objective The present study was designed to explore response variability and its correlation with recurrent cardiovascular events in Chinese patients undergoing Percutaneous coronary intervention (PCI).

Methods Platelet aggregation (5 and $20 \mu \mathrm{mol} / 1, \mathrm{ADP}$ ) and the expression of CD $62 \mathrm{p}$ (P-selectin) and CD $42 \mathrm{~b}$ (GP Ib) were measured at baseline, at $12 \mathrm{~h}$, and at $36 \mathrm{~h}$ after clopidogrel loading dose in 111 consecutive Chinese patients undergoing PCI. Clopidogrel responsiveness was defined according to the degree of inhibition of platelet function (platelet aggregation and P-selectin expression) after clopidogrel administration compared with the baseline values (before clopidogrel), IPA $<10 \%$ (clopidogrel non-responders), 10\% $\leq 30 \%$ (responders). Patients were followed up in 1, 3, 6, 12 months after PCI. The study end points was defined recurrent cardiovascular events (recurrent CV: cardiovascular death, stent thrombosis, ischaemic stroke, ACS), readmission, bleeding events.

Results There was marked interindividual variability in drug response, as measured by platelet aggregation and P-selectin expression. The ratios of the non-responders at 12 and $36 \mathrm{~h}$ were $32 \%(35 / 109)$ and $19 \%(21 / 109)$, respectively, by $5 \mu \mathrm{mol} / 1$ ADP; 38\% (41/109) and 28\% (31/109)by $20 \mu \mathrm{mol} / 1 \mathrm{ADP}$; and $27 \%(29 / 109)$ and $17 \%(19 / 109)$ by P-selectin expression. The maximal aggregation rates stimulated by $5 \mu \mathrm{mol} / 1$ ADP of nonresponders were significantly higher compared with those of the responders $(57.53 \pm 14.24 \%$ vs $33.91 \pm 10.79, p<0.0001)$ at $12 \mathrm{~h}$ and at $36 \mathrm{~h}(48.65 \pm 15.46$ vs $30.31 \pm 16.04, \mathrm{p}<0.0001)$. During the 12 -month follow-up, 21(19.63\%) patients recurrent cavascular events occurred: 5 deaths $(6.47 \%), 2$ ischaemic stroke (1.87\%), 14(19.63\%)ACS. Cumulative recurrent CV in non-responders was significantly higher than responders in 3 months ( $p=0.005), 6$ months $(p=0.002), 12$ months $(p<0.0001)$. Multivariable Cox regression analysis, including pertinent covariables, confirmed individual responsiveness variability to clopidogrel as a significant independent predictor of 12 -month recurrent CV. Non-responders carried a 24.28 -fold risk $(95 \%$ CI 3.05 to $193.41, p=0.003$ ) compared with responders. Low responders carried 11.95 -fold risk (95\% CI 3.08 to 178.46 , $\mathrm{p}=0.002)$ compared with responders. During the 12 -month follow-up, 61(57.01\%) patients occurred readmission, nonresponders had a higher incidence of readmission $(p=0.01)$ than responders. The bleeding scores in responders were significantly higher than non-responders during $3(p=0.026)$, $6(p=0.040), 12(p=0.031)$ months follow-up by Bleed Score classification.

Conclusion The antiplatelet effectiveness of clopidogrel has a wide interindividual variation among Chinese patients undergoing PCI. Non-responders' IPA after clopidogrel is higher than that of responders. Individual responsiveness variability to clopidogrel is a significant independent predictor to recurrent cardiovascular events, non-responders and low responders to clopidogrel have higher risk for recurrent cardiovascular events than responders. 